\title{
EDITORIAL
}

\section{Health outcomes assessment and economic evaluation in COPD: challenges and opportunities}

\author{
S.D. Sullivan*, A.S. Buist", K. Weiss"
}

\begin{abstract}
As a leading and growing cause of mortality and morbidity worldwide, chronic obstructive pulmonary disease (COPD) is now widely recognised as a major global public health problem with substantial medical and economic burdens. A decade ago, the annual per capita expenditure for COPD treatment in the USA was $\$ 8,482$, more than twice that for people without COPD [1]. With an increasingly older population in most countries, the prevalence of COPD and its associated healthcare costs will grow. In a disturbing report, the Global Burden of Disease Study concluded that from 1990-2020, COPD would increase worldwide from the sixth- to the third-leading cause of death and from the twelfth- to the fifth-leading cause of disability-adjusted life years lost [2].
\end{abstract}

To develop a coordinated international strategy for diagnosing and managing COPD, the National Heart, Lung and Blood Institute in conjunction with the World Health Organization, organised the Global Obstructive Lung Disease Initiative. This programme's objectives were to enhance awareness among public health officials, the medical community and the public, concerning the mounting health problems associated with COPD, to promote studies investigating the causes for increasing COPD prevalence and to implement programmes for COPD prevention [3].

Partly because of the emerging public health concern, there is now an acute interest in COPD and in defining treatments and treatment strategies that make a difference in the outcomes of patients. Unfortunately, there is some disagreement concerning optimal outcomes of care in this population. Regulatory authorities require lung function measurements for licensing of new products, whereas clinicians and patients are more interested in symptoms, functioning and health status. Payers and reimbursement authorities want to know whether new treatments will provide better outcomes at a reasonable price. In spite of this, little attention has been given to the issues and challenges that face health outcomes' assessments and economic evaluations in COPD.

Health outcomes' research focuses on clinical and patientreported outcome measures that assess lung function as well as symptoms, functional status, treatment preferences, satisfaction and patients' quality of life. Organisations that provide payment for healthcare services are under increasing pressure to consider health outcomes along with the economic consequences of treatment when making decisions regarding formulary inclusions and reimbursement.

\footnotetext{
*University of Washington, Seattle, WA, ${ }^{\text {*}}$ Oregon Health Sciences University, Portland, OR and "Midwest Center for Health Services and Policy Research, Hines VA Medical Center and Division of General Medicine, Dept of Medicine, Northwestern University Feinberg School of Medicine, Chicago, IL, USA.

Correspondence: S. Sullivan, Dept of Pharmacy and Health Services, Box 357630, University of Washington, Seattle, WA 98195-7630, USA. Fax: 1 2066858153. E-mail: sdsull@u.washington.edu
}

\section{Invited symposium on health outcomes and economic evaluation}

An invited symposium of experts was organised to review, critically evaluate and discuss the methodological and application issues surrounding health outcomes and economic evaluation, within the clinical context of COPD and to explore the substantial advances that have been made in the definition, constructs, tools and acceptance of COPD in recent years. Underscoring the importance of such a gathering was the fact that there has not been a conference or symposium in recent memory that attempted to bring together experts in the disciplines of clinical research, health outcomes' measurement and economic evaluation.

The symposium, comprising a panel of 23 COPD experts (see below) in healthcare outcomes, convened in Paris, France, from April 29-May 1 2001. Panelists reviewed four different topics related to outcome measurements in COPD: 1) epidemiology and natural history; 2) physiological outcome measures; 3) functional status and quality of life; and 4) burden of disease and economic evaluation. Following each expert's presentation of a paper, another expert offered a formal reaction, which was subsequently followed by a discussion involving the entire panel. The presentations on physiological outcomes, functional outcomes, exacerbations and burden of disease were each followed by a 3-h breakout discussion. The articles that appear in this journal supplement are based on the authors' presentations enhanced by the contributions from the panel discussion.

\section{Epidemiology and natural history}

S.T. Weiss and D.S. Postma each have $>25 \mathrm{yrs}$ of experience treating patients with lung disease. S.T. Weiss has a strong interest in the risk factors, genetics and natural history of airway diseases, asthma and COPD. D.S. Postma has been involved in various aspects of COPD management, with an emphasis on epidemiology and the basic science of COPD. In their review, they explore the major obstacles to COPD diagnosis related to testing and disease course. They examine the discrepancy between patient symptom reports and objective spirometry measurements, as well as other obstacles to COPD diagnosis, including the reluctance among many patients to report respiratory complaints, the failure to identify childhood asthma and airway hyperresponsiveness and a tendency among clinicians to diagnose males with COPD and females with asthma or asthmatic bronchitis. Indeed, the prevalence of COPD is higher among males than females. This gap continues to narrow, however, because of the increased incidence of smoking in females. Additionally, females may be more sensitive to the detrimental effects of cigarette smoke on pulmonary function, possibly because of 
reduced airway size or enhanced airway hyperresponsiveness compared with males.

COPD is a heterogeneous disease, with symptoms and pulmonary function varying considerably among patients. Even so, the authors point out that COPD is defined physiologically, based on pulmonary testing with little emphasis on the inflammatory aspects of the disease. COPD lacks intermediate phenotypes that can be tracked and patients who are often $\geqslant 60$ yrs of age have comorbidities, such as cancer or heart disease, which can alter the course of COPD and natural history, further complicating diagnosis and measurement. The authors suggest that the future value of COPD research will be influenced by the ability to clarify the diagnosis, risk factors, sex differences and comorbidities associated with the disease.

\section{Clinical measures}

\section{Methodological issues}

In assessing disease progression and the value of treatment, the appropriate selection of an outcome measure in COPD is critical. P.W. Jones and R.M. Kaplan examine the major methodological issues related to COPD outcome selection, measurement and statistical evaluation. P.W. Jones focuses on measuring health status in patients with COPD and R.M. Kaplan has been involved with outcome studies in COPD for 20 yrs. These experts note that outcome measures in COPD typically assess changes in some disease-related biological factor, such as pulmonary functioning, but from the patients' perspective, health-related outcomes that improve routine daily functioning, symptoms and quality of life are considered the primary goals of COPD treatment. Although not always correlated, both sets of outcome measures are equally important in evaluating the effectiveness of COPD treatment. Furthermore, the authors describe two broad categories of outcome measures used in respiratory medicine: specific and global. A specific outcome measure assesses a single biological variable, such as forced expiratory volume in one second (FEV1), and ideally, the choice of a specific outcome variable should be related to the study's purpose or the treatment's mechanism of action. In contrast, global outcomes attempt to quantify the overall therapeutic actions of an intervention and may be more sensitive to clinically significant changes in the patient's health status over time. In COPD, examples of a global measure include exercise tolerance and health status measured by questionnaires, such as the Short Form (SF)-36 and the Sickness Impact Profile. The authors also explore the indispensable qualities or criteria for an outcome measure in COPD: validity, reliability, repeatability and sensitivity. Currently, clinical outcome measures, such as breathlessness, exercise capacity and health status, come closest to meeting these criteria.

\section{Physiological outcome measures}

In the absence of treatment that alters the fundamental disease process in COPD, the focus of therapy and of outcome measures has shifted to improving the patient's health-related quality of life. In this setting, traditional spirometry measures perform poorly in that they are not well correlated with quality-of-life measures. R.O. Crapo and F.E. Hargreave explore alternatives to conventional FEV1 and FEV1/forced vital capacity outcome measurements in COPD. R.O. Crapo has had a career-long interest in the measurement of pulmonary function and F.E. Hargreave is a clinical researcher in airway disease with a recent interest in the use of noninvasive measures to assess airway inflammation, particularly induced sputum. Peak flow, mid-flows, inspiratory capacity (IC) and partial expiratory flow volume (PEFV) are proposed as alternatives to standard spirometry. Even though IC and PEFV are better correlated with symptomatology and quality of life, they are used infrequently. Other physiological tests, timed walk distance and the endurance shuttle-walk test, also display a greater correlation with quality-of-life measures when compared with FEV1. Additionally, improving the treatment of COPD requires a clear understanding of the inflammatory process involved, an insight not provided by spirometry. The analysis of inflammatory markers in sputum has become easier and more reliable in recent years and the authors suggest that sputum analysis will help predict which patients will respond to corticosteroid therapy. In COPD, the hallmark sputum change is neutrophilia, although eosinophilia is present in some patients.

\section{Exacerbations}

Another important, but often overlooked, parameter of COPD is the occurrence of exacerbations; generally a sustained, acute, worsening of disease that requires a change in regular medication. Although exacerbations only affect $\sim 20 \%$ of patients with moderate-to-severe COPD, they incur a substantial healthcare burden and thus require early recognition, diagnosis and treatment. S. Burge has had a primary interest in occupational lung diseases, specifically the treatment of COPD. Along with S. Burge, J.A. Wedzicha, one of the worldwide distinguished experts in the area of COPD exacerbations, examined the challenges in defining, classifying and managing COPD exacerbations. These authors point out that the definition and diagnosis of COPD exacerbations are daunting because physiological changes are often small and variable. Moreover, COPD exacerbations may be triggered by bacterial and viral infections, pollution, cold weather and interruption in treatment. In clinical trials, exacerbation frequency (the reciprocal of interexacerbation interval) and severity (mild: managed at home; moderate: requiring corticosteroid; severe: requiring hospitalisation) are useful classifications but difficult to measure.

Exacerbation frequency varies by season in many climates and is related to influenza and other viral outbreaks, thus, studies must account for seasonal variations when using exacerbation frequency as an outcome variable. A retrospective diagnosis of COPD exacerbation can be made using information from health-related databases, but it may be difficult to ascertain when one exacerbation ends and another begins, since relapses are common after severe exacerbations. The authors suggest that patient daily diary cards can be useful in discerning exacerbation duration.

The authors also explore strategies for COPD exacerbation management through the use of influenza and pneumococcal vaccinations, regular inhaled corticosteroids, mucolytics and antibiotic therapy during an exacerbation.

\section{Functional status and quality of life}

An important treatment goal in COPD is reducing its personal and social burden by improving symptoms, functional status and quality of life. There is a large number of instruments available to measure these patient-reported, health-status outcomes and clinicians should understand their strengths and weaknesses. J.R. Curtis and D.L. Patrick 
examine the appropriateness of various instruments for measuring quality-of-life changes in patients with COPD. These authors, who have worked on health-status and qualityof-life issues in chronic lung disease, describe measures that are sensitive to general health status as well as those that are disease-specific. Typically, these instruments have established reliability (reproducible results), validity (measures what it purports to measure) and responsiveness (ability to detect changes over time). In COPD, general health may be evaluated reliably using the Sickness Impact Profile or the SF-36. Disease-specific, health-status measures include the Chronic Respiratory Disease Questionnaire and the St George's Respiratory Questionnaire.

\section{Burden of disease and economic evaluation}

In today's cost-conscious healthcare environment, understanding the economic implications of COPD and its treatment can be as important as understanding its clinical impact. S.D. Ramsey, a general internist and health economist, and S.D. Sullivan examine the types and relative strengths of economic evaluation studies that can be used in COPD research.

There are two general types of cost studies. Burdenof-illness studies are designed to estimate the economic impact of a disease on a whole society (e.g. nation) in monetary terms. Economic evaluation studies, or costeffectiveness analyses (CEAs), are designed to examine the incremental economic impact of a particular versus an alternative therapy. The attributes important in all cost evaluation studies are described. For instance, all economic studies of COPD must include an assessment of the direct medical cost (all medical goods and services used in treatment), the direct nonmedical costs (nonmedical items related to patient care, such as caregiver expenses and transportation) and productivity costs (lost wages). Additionally, in cost studies, all anticipated costs should be projected and tracked. Often in COPD, the timeline for tracking is a lifetime. Guidelines developed for conducting CEAs emphasise the need to define several parameters important for accurate data analysis and interpretation, such as study perspective (usually that of the healthcare provider), the cohort under study, the time horizon used and the standard reference treatments for the intervention under study, as well as the discounting or estimating process used to project COPD-related costs in future years. In cost analyses, S.D. Ramsey and S.D. Sullivan advocate the inclusion of quality-of-life measures meaningful to the patient, clinician and insurer. For example, quality-adjusted life years are useful and well studied in this context.

The authors of the articles in this supplement have identified and clarified many of the important health outcomes and economic evaluation issues and challenges associated with the diagnosis and management of chronic obstructive pulmonary disease. However, this should only be considered as the first step toward defining the appropriate role for health outcomes and economic evaluations in chronic obstructive pulmonary disease.

\begin{abstract}
Symposium participant list: Canada: F.E. Hargreave, Firestone Institute for Respiratory Health, McMaster University, Hamilton, ON; S. Suissa, Montreal, QC; Denmark: J. Vestbo, Hvidovre; The Netherlands: H. Kerstjens, Groningen; D.S. Postma, Groningen University Hospital, Groningen; M. Rutten-van Mölken, Rotterdam; Norway: A. Gulsvik, Bergen; Spain: R. RodriguezRoisin, Barcelona; Sweden: C-G. Löfdahl, Lund; UK: S. Burge, Dept of Respiratory Medicine, East Birmingham Hospital, Birmingham; P.W. Jones, St George's Hospital Medical School, London; S. Spencer, London; J.A. Wedzicha, St Bartholomew's Hospital, London; USA: S. Buist (Co-Chair), Portland, OR; R.O. Crapo, Latter Day Saints Hospital, University of Utah, Salt Lake City, UT; J.R. Curtis, Division of Pulmonary and Critical Care Medicine, University of Washington, Seattle, WA; R.M. Kaplan, Chair of Family and Preventative Medicine, University of California, San Diego, CA; D.L. Patrick, Head of the Social and Behavioral Sciences Programme, School of Public Health, University of Washington, Seattle, WA; S.D. Ramsey, Fred Hutchinson Cancer Research Center and the Dept of Medicine, University of Washington, Seattle, WA; S.D. Sullivan (CoChair), Director of the Pharmaceutical Outcomes Research and Policy Programme, University of Washington, Seattle, WA; W.M. Vollmer, Portland, OR; K. Weiss (Co-Chair), Chicago, IL; S.T. Weiss, Head of the Respiratory and Genetic unit, Channing Laboratory, Brigham \& Women's Hospital, Boston, MA.
\end{abstract}

\section{References}

1. Global Initiative for Chronic Obstructive Pulmonary Disease. Burden of COPD. http://www.goldcopd/workshop/ ch2.html. May 2000. Accessed May 3, 2002.

2. Gulsvik A. The global burden and impact of chronic obstructive pulmonary disease worldwide. Monaldi Arch Chest Dis 2001; 56: 261-264.

3. Hurd S. The impact of COPD on lung health worldwide: epidemiology and incidence. Chest 2000; 117: Suppl. 2, 1S-4S. 